\title{
Enhanced recovery (ERAS) protocol in patients undergoing laparoscopic total gastrectomy
}

\author{
Michał Pędziwiatr ${ }^{1,2}$, Maciej Matłok ${ }^{1,2}$, Mikhail Kisialeuski ${ }^{1,2}$, Piotr Major ${ }^{1,2}$, Marcin Migaczewski $^{1,2}$, Piotr Budzyński ${ }^{1,2}$, \\ Sebastian Ochenduszko ${ }^{3}$, Kazimierz Rembiasz ${ }^{1}$, Andrzej Budzyński ${ }^{1,2}$ \\ ${ }^{1} 2^{\text {nd }}$ Department of General Surgery, Jagiellonian University Medical College, Krakow, Poland \\ ${ }^{2}$ Department of Endoscopic, Metabolic and Soft Tissue Tumors Surgery, University Hospital, Krakow, Poland \\ ${ }^{3}$ Department of Oncology, University Hospital, Krakow, Poland
}

Videosurgery Miniinv 2014; 9 (2): 252-257

DOI: $10.5114 /$ wiitm.2014.43076

\begin{abstract}
Introduction: Laparoscopic technique combined with the ERAS (Enhanced Recovery after Surgery) protocol enables a shorter hospital stay and a lower complication rate. Although it has been widely used in many patients undergoing elective abdominal surgery, especially in patients with colorectal cancer, there are only a few papers describing laparoscopic total gastrectomy and the enhanced recovery protocol in patients with gastric cancer. Minimally invasive gastrectomy is still an uncommon procedure, mostly because of its difficulty.

Aim: To present the preliminary results of treatment of patients with gastric neoplasms who underwent laparoscopic gastrectomy D2 with perioperative care according to ERAS principles.

Material and methods: Eleven patients (5 male and 6 female, age 52-77 years) underwent laparoscopic D2 gastrectomy with intracorporeal esophagojejunal anastomosis. In all patients the ERAS protocol was implemented. We analyzed operation time, complications and hospital stay. Additionally we focused on operative technique as well as the perioperative care protocol.

Results: The mean duration of the procedure was $245 \mathrm{~min}$. There was 1 conversion due to unclear tumor infiltration. Mean hospital stay was 4.6 days. One postoperative complication (central venous catheter sepsis) was reported. Histological analysis confirmed the tentative diagnosis (RO resection) in 10/11 patients. There were no readmissions. Conclusions: Laparoscopic gastrectomy is a valuable alternative to the classical approach and combined with the ERAS protocol can result in reduced hospital stay. However, due to the small group of patients it is difficult to adequately assess the incidence of early and late complications of the laparoscopic procedures; therefore further research is needed.
\end{abstract}

Key words: laparoscopy, postoperative care, gastrectomy, fast-track, enhanced recovery.

\section{Introduction}

An increase in the number of laparoscopic operations performed due to gastrointestinal neoplasms can be noticed [1]. Laparoscopy in comparison to the classical approach is associated with a lesser degree of postoperative pain, quicker recovery and superior life quality in the postoperative period [2]. Combined with the ERAS (Enhanced Recovery after Surgery) protocol, laparoscopy leads to a shorter hospital stay and decreased risk of postoperative complications, especially in case of colorectal surgery [3]. The first reports on laparoscopic gastric resections due to cancer appeared in the early 1990 s $[4,5]$. Simi- 
lar to the operations on other organs, laparoscopic gastrectomy is associated with better postoperative outcomes when compared to the classical approach [6]. Even though such procedures were performed over 20 years ago for the first time, there are relatively few centers in the world where laparoscopy is routinely used for gastric resection. Publications analyzing long-term results of gastric cancer treatment by this technique are also not numerous [7]. The first laparoscopic gastrectomy in Poland was performed in 2012 by Dr Maciej Michalik and his surgical team. This kind of laparoscopic surgery is performed only in a few centers in our country and such procedures are not routine practice.

\section{Aim}

The aim of the study is to present early results of treatment of patients with gastric neoplasms who underwent laparoscopic gastrectomy and in whose perioperative period the ERAS protocol was used.

\section{Material and methods}

Prospective analysis of patients who underwent laparoscopic D2 gastrectomy in the period from January to December 2013 was performed. The studied group included 11 patients ( 6 females and 5 males). Mean age was 59.8 years (40-77 years) and mean body mass index was $23.4 \mathrm{~kg} / \mathrm{m}^{2}\left(16.1-39.1 \mathrm{~kg} / \mathrm{m}^{2}\right)$.

The indications for the surgery were malignant gastric tumors: adenocarcinoma in 10 patients and multiple neuroendocrine tumor in 1 patient. In all patients prior to the surgery the diagnosis was confirmed with the histopathological result and the imaging studies did not show signs of distant metastases. Seven out of 10 patients received neoadjuvant chemotherapy due to the preoperative state of advancement.

Early treatment results, such as number of conversions, number and character of perioperative complications, postoperative period and the length of hospital stay, were then analyzed.

\section{Operative technique}

After creation of pneumoperitoneum 5 trocars were introduced. Trocar sites were infiltrated with bupivacaine solution. The procedure began with dissection of the gastric cardia and resection of the gastohepatic ligament with excision of pericardial lymph nodes $\left(1^{\text {st }}\right.$ and $2^{\text {nd }}$ gastric lymph node stations), followed by mobilization of the distal, mediastinal part of the esophagus. In the next step, the greater omentum was divided from the transverse colon and the omental sac was opened. After cutting the spleno-transversal ligament the fatty tissue of the splenic hilum and that superior to the splenic artery were removed together with lymph nodes of the $10^{\text {th }}$ and $11^{\text {th }}$ gastric lymph node stations. The left gastric artery was dissected, clipped and divided at the point where it leaves the celiac trunk, with subsequent removal of lymph nodes ( $7^{\text {th }}$ gastric lymph node station) and fatty tissue of that area. The esophagus was transected with an Echelon 45 stapler (blue color). The right gastric artery was then dissected, clipped and divided at the point where it leaves the common hepatic artery and the lymph nodes of the $8^{\text {th }}$ lymph node station were removed. The duodenum was isolated and cut and the duodenal stump was closed with an Echelon 60 stapler (white color). Subsequently, a retrocolic esophagoenteric anastomosis with $70 \mathrm{~cm}$ long Roux loop was created. The posterior $2 / 3$ of the anastomosis was performed with an Echelon 45 stapler (white color), and its anterior wall was closed with two absorbable continuous 3/0 stitches. Enteroenteric anastomosis was performed with a similar technique (Echelon 45 stapler (white color) and two absorbable continuous $3 / 0$ stitches). The specimen was placed in a plastic bag and then removed through minilaparotomy protected with the Alexis wound protector. The blue dye leakage test of the esophagoenteric anastomosis was performed at the end of surgery. Drains (used only in 5 patients) were removed on the day after surgery. In two patients drains were removed later because during the intraoperative blue dye leakage test there was a leakage and a second layer of sutures had to be put over the stapling line. In the case of one patient due to the presence of a single lesion in the duodenal bulb, the margins of the distal resection were widened. In another patient, because of the localization of cancer, it was necessary to dissect the larger part of the esophagus for a satisfactory proximal resection margin. For better postoperative pain management transversus abdominis plane block (TAP-block) was performed at the end of the procedure.

\section{Perioperative care}

Patients were admitted to the hospital 1 day before the surgery. At admission, detailed information 
on planned treatment and perioperative care was provided to them. Two hours before the surgery all patients received $250 \mathrm{ml}$ of carbohydrate-rich drink. Intraoperatively the fluid therapy was restricted, and sodium-rich crystalloids were partially replaced by colloids. All patients received prophylaxis for postoperative nausea and vomiting by intravenous dexamethasone and ondansetron administration before the end of surgery. No esophagoenteric tubes were left after the surgery. On the day of surgery patients could drink clear fluids as desired. Intravenous administration of fluids was restricted. All patients were mobilized (sat up in bed, walked with assistance) on the same day as the operation. On the first postoperative day a single shot of antibiotics was administered (2 g of cefazolin). Ketoprofen and paracetamol were used for analgesia. Only in 3 patients was there a need to administer low subcutaneous morphine doses (not more than $20 \mathrm{mg}$ per day). Esophagojejunal anastomosis was tested for patency or leakage via upper gastrointestinal tract $X$-ray series with water-soluble contrast. None of the patients had stenosis or anastomotic leakage. The diet was expanded gradually with supplementation of protein-rich drinks. Drains were removed on the second day.

\section{Results}

Mean operative time was $245 \min$ (160-420 min). In 1 patient due to cancer infiltration of the transverse colon a conversion was performed and the resection margins included a segment of colon. In another patient with infiltration on the pancreas hand-assisted $\mathrm{R} 2$ resection was performed (hand-assisted laparoscopic gastrectomy - HALG). Moreover, in one patient splenic artery injury happened during dissection of neighboring tissues with a Ligasure vessel sealer. The vessel was successfully sutured, but due to the suturing delay and signs of thrombosis in the vessel, laparoscopic splenectomy had to be performed. There were no other intraoperative complications. In 1 of the patients who preoperatively received parenteral nutrition for 2 weeks central venous catheter sepsis occurred. It was successfully managed with antibiotic therapy.

All of the patients (100\%) started postoperative oral fluid intake on the day of surgery. After the test of esophagojejunal anastomosis for patency via upper gastrointestinal tract $\mathrm{X}$-ray series with water-sol- uble contrast, the diet was extended. Even though there were no postoperative nausea and vomiting episodes, full diet and oral protein supplements on the second postoperative day were tolerated well only in 8 out of 11 (72.7\%) patients. All of the patients (100\%) were mobilized on the day of surgery (sitting up, walking), and on the second postoperative day every patient spent at least $5 \mathrm{~h}$ out of bed. The urinary catheter was removed in the evening of the day of surgery. No urinary retention was observed. In 2 patients (18.1\%) opioid administration was needed. Mean length of hospital stay was 4.6 days. No rehospitalizations within 30 days after discharge were noted.

Histological analysis confirmed the tentative diagnosis in all patients. Besides the aforementioned patient with cancer infiltration of the pancreas, RO resection was achieved. Every specimen included lymph nodes from the D2 group. All patients were directed to the oncological outpatient clinic for further adjuvant therapy.

\section{Discussion}

Even though the first laparoscopic gastrectomies due to cancer were performed in 1993, there are controversies regarding this method [8]. Opponents of this technique question the oncological quality of the procedure compared to the classical approach. Comparison of laparoscopic and classical methods concluded that the number of lymph nodes removed during the operation is similar regardless of preferred technique [9-11]. Histopathological evaluation in all abovementioned operations proved them to be radical, and lymphangiectomy included lymph nodes from the D2 group. It also seems worth mentioning that the recent meta-analyses did not show improvement of long-term treatment results when lymphangiectomy was extended to D2. Moreover, D2 gastrectomy (as well as D3) was associated with higher risk of early postoperative complications and greater mortality when compared to D1 [10, 12-14].

Undoubtedly, laparoscopic gastrectomy is one of the most difficult operations among the minimally invasive abdominal procedures and the success rate and the lymphangiectomy range strongly depend on the surgeon's experience. There are nearly 400 advanced laparoscopic procedures performed yearly in our hospital. A substantial number of them include procedures on the stomach, and the experience ac- 
quired during bariatric procedures (gastric bypass, sleeve gastrectomy), operations on the gastroesophageal junction (Nissen fundoplication, Heller's cardiomyotomy), splenectomies, and most importantly, operations due to gastrointestinal stromal tumors (GIST), allowed the first total gastrectomy due to malignancy, preserving oncological radicality of the procedure, to be performed. The results of available research studies indicated that the surgical center's experience in gastric resections has a significant impact on the results of the treatment and 5-year survival [14]. Thus the extension of the procedure seems to have a smaller influence on the treatment results in gastric cancer patients. When performing laparoscopic gastrectomy we decided to perform an intracorporeal esophagojejunal anastomosis with the Roux loop according to the same technique used in our center during bariatric gastric bypass procedures. Although there are other methods of anastomotic creation, based on our experience and opinions of other authors this exact method seems to be associated with lower probability of leakage or stenosis [15-17].

Perioperative care was based on the guidelines of the ERAS protocol, which we actively use in our center in colorectal and bariatric surgery. The use of the ERAS protocol aims to achieve quicker recovery after surgery and together with laparoscopy can lead to a decrease of early complications [6]. Although fasting even up to $12 \mathrm{~h}$ prior to the surgery has been standard practice to avoid pulmonary aspiration in elective surgery, a review of recent studies found no scientific support for this. A review of available studies and clinical observations provided robust evidence that reducing the preoperative fasting for clear fluids to $2 \mathrm{~h}$ and solid foods to $6 \mathrm{~h}$ does not lead to an increase in complication rates. Additionally, administration of carbohydrate-rich fluids $2-3 \mathrm{~h}$ prior to the surgery reduces the fasting time, which decreases catabolic reaction, reduces insulin resistance of the organism, and has an overall positive influence on the nitrogen balance $[18,19]$. It is also proved to be safe in patients with uncomplicated type 2 diabetes mellitus [20]. In the postoperative period a liquid diet can be applied on the day of surgery. From the second day, after radiological control of esophagoenteric anastomotic sufficiency, the diet is gradually enriched, so on the third postoperative day patients received a full mixed semi-liquid diet. The majority of patients in our study (72\%) tolerated it well.
In many centers postoperative oral intake tends to be restrictive; however, there is strong evidence that early postoperative enteral feeding (on the day of surgery) reduces the catabolic phase of the organism, fastens the return of intestinal function and decreases the risk of postoperative complications (as well as reducing the risk of anastomotic leakage). This was most extensively studied in colorectal surgery $[21,22]$.

Without doubt, there are far fewer publications available on the similar topic in the case of upper gastrointestinal tract surgery. According to Lassen et al., who performed a multicenter randomized control trial on upper gastrointestinal surgery patients, early introduction of a full diet from the first postoperative day was not associated with an increased complications rate and allowed the hospital stay to be reduced [23]. Summing up, it seems that early oral intake, starting with a small amount of mixed food or protein-rich drinks, results in a faster return of the gastrointestinal tract and reduced hospital stay length.

The key element of modern perioperative care is optimal fluid management during and after surgery. Excessive fluid administration leads to water accumulation in the extravascular compartment and results in tissue edema, which is detrimental to the surgical outcome. This will affect intestinal function and prolong the postoperative return of peristalsis [24]. Moreover, overload with crystalloids significantly increases the complications rate, including anastomotic dehiscence $[25,26]$. To avoid this, restrictive fluid therapy is essential during the operation, and when possible, intravenous fluid therapy should be limited and crystalloids should be replaced with colloids. Early enteral feeding allows one to reduce postoperative intravenous fluid administration. The examined group of patients was allowed to drink desired amounts of fluids on the day of the surgery, which partially eliminated the need for intravenous drips. No advantage of routine use of an esophagoenteric tube has been shown. It did not decrease the anastomotic dehiscence risk, the number of pulmonary complications or the mortality rate. Additionally it has been shown that leaving the esophagoenteric tube prolongs postoperative ileus and time of the first passage of flatus. Also it significantly decreases the patient's comfort postoperatively [27]. Evidence also shows no benefit in use of drains in the case of colorectal surgery, in surgery of the pancreas, ap- 
pendix or gallbladder $[28,29]$. No publications on this topic were found in regards to upper gastrointestinal surgery. An advantage of minimally invasive technique can be that relaparoscopy in the case of suspicion of bleeding or anastomotic dehiscence is associated with lower complications risk than relaparotomy [6].

In the described group of patients drains were left in situ in the first 3 patients as well as the 2 aforementioned patients who had a positive intraoperative blue dye leakage test and required an additional layer of stitches over the stapling line. However, minimal postoperative drainage allowed for the early removal of drains.

A fundamental role in postoperative care is dedicated to analgesia. Even though epidural anesthesia plays the most important role in classical operations, its use in laparoscopy is limited. Evidence has shown that analgesia based on non-steroidal anti-inflammatory drugs is generally sufficient in laparoscopic surgery [30, 31]. In our opinion non-steroidal anti-inflammatory drugs together with paracetamol can provide full pain control in the first few days after surgery. When needed, small doses of stronger painkillers can be administered at the patient's will. An important element of ERAS protocol-based postoperative care is early mobilization [32]. The importance of early mobilization is well depicted by the study conducted in Yeovil, which showed that failure of early mobilization is one of the most common causes of deviation from the ERAS protocol and is associated with prolonged hospital stay [33]. Early effective postoperative mobilization of patients is possible mainly due to adequate pain control. Similarly, restriction of intravenous fluid administration, early urinary catheter and drain removal improves postoperative patients' comfort, leading to earlier postoperative mobilization.

The presented results do not allow us to conclude about long-term treatment results in patients who underwent laparoscopic gastrectomy. However, the recently performed studies suggest that minimally invasive techniques allow for similar long-term results when compared to the classical approach [7, $34,35]$. Laparoscopic operations are associated with smaller blood loss and a lower complication rate. They are characterized by shorter hospital stay, and their combination with the ERAS program results in faster recovery, which is especially valuable in the context of adjuvant chemotherapy planned in the postoperative period.

\section{Conclusions}

Laparoscopic gastrectomy performed in a high volume center for minimally invasive surgery could be a valuable alternative to the classical approach. Even though the laparoscopic method is more technically demanding and more time-consuming, combined with the ERAS protocol it can result in a reduced hospital stay. Due to the small group of patients it is difficult to adequately assess the incidence of early and late complications of the laparoscopic procedures, although some publications confirm the abovementioned advantages of this method, emphasizing the possibility of oncological radicality in gastric cancer surgery.

\section{Acknowledgments}

This publication was financed with the support of Leading National Research Centre 2012-2017.

\section{References}

1. Matsuhashi N, Osada S, Yamaguchi K, et al. Oncologic outcomes of laparoscopic gastrectomy: a single-center safety and feasibility study. Surg Endosc 2013; 27: 1973-9.

2. Adachi Y, Suematsu T, Shiraishi N, et al. Quality of life after laparoscopy-assisted Billroth I gastrectomy. Ann Surg 1999; 229: 49-54.

3. Gustafsson UO, Scott MJ, Schwenk W, et al. Guidelines for perioperative care in elective colonic surgery: Enhanced Recovery After Surgery (ERAS $\left.{ }^{\circledR}\right)$ Society recommendations. World J Surg 2013; 37: 259-84.

4. Goh P, Tekant Y, Isaac J, et al. The technique of laparoscopic Billroth II gastrectomy. Surg Laparosc Endosc 1992; 2: 258-60.

5. Kitano S, Iso Y, Moriyama M, et al. Laparoscopy-assisted Billroth I gastrectomy. Surg Laparosc Endosc 1994; 4: 146-8.

6. Grantcharov TP, Kehlet H. Laparoscopic gastric surgery in an enhanced recovery programme. Br J Surg 2010; 97: 1547-51.

7. Lee J, Lee C, Son S, et al. Laparoscopic versus open gastrectomy for gastric cancer: Long-term oncologic results. Surgery 2013; 12: S0039-6060.

8. Azagra JS, Goergen M, De Simone P, et al. Minimally invasive surgery for gastric cancer. Surg Endosc 1999; 13: 351-7.

9. Du J, Zheng J, Li Y, et al. Laparoscopy-assisted total gastrectomy with extended lymph node resection for advanced gastric cancer: reports of 82 cases. Hepatogastroenterology 2010; 57 : 1589-94.

10. Cui M, Xing JD, Yang W, et al. D2 dissection in laparoscopic and open gastrectomy for gastric cancer. World J Gastroenterol 2012; 18: 833-9

11. Wei HB, Wei B, Qi CL, et al. Laparoscopic versus open gastrectomy with D2 lymph node dissection for gastric cancer: a meta-analysis. Surg Laparosc Endosc Percutan Tech 2011; 21: 383-90. 
12. Cuschieri A, Fayers P, Fielding J, et al. Postoperative morbidity and mortality after D1 and D2 resections for gastric cancer: preliminary results of the MRC randomised controlled surgical trial. The Surgical Cooperative Group. Lancet 1996; 347: 995-9.

13. McCulloch P, Niita ME, Kazi H, et al. Gastrectomy with extended lymphadenectomy for primary treatment of gastric cancer. $\mathrm{Br}$ J Surg 2005; 92: 5-13.

14. McCulloch P, Nita ME, Kazi $H$, et al. Extended versus limited lymph nodes dissection technique for adenocarcinoma of the stomach. Cochrane Database Syst Rev 2004; 4: CD001964.

15. Okabe H, Obama K, Tsunoda S, et al. Advantage of completely laparoscopic gastrectomy with linear stapled reconstruction: a long-term follow-up Study. Ann Surg 2014; 259: 109-16.

16. Okabe H, Obama K, Tanaka E, et al. Intracorporeal esophagojejunal anastomosis after laparoscopic total gastrectomy for patients with gastric cancer. Surg Endosc 2009; 23: 2167-71.

17. Kim JJ, Song KY, Chin HM, et al. Totally laparoscopic gastrectomy with various types of intracorporeal anastomosis using laparoscopic linear staplers: preliminary experience. Surg Endosc 2008; 22: 436-42.

18. Nygren J, Soop M, Thorell A, et al. Preoperative oral carbohydrate administration reduces postoperative insulin resistance. Clin Nutr 1998; 17: 65-71.

19. Svanfeldt M, Thorell A, Hausel J, et al. Randomized clinical trial of the effect of preoperative oral carbohydrate treatment on postoperative whole-body protein and glucose kinetics. $\mathrm{Br}$ J Surg 2007; 94: 1342-50.

20. Breuer JP, von Dossow V, von Heymann C, et al. Preoperative oral carbohydrate administration to ASA III-IV patients undergoing elective cardiac surgery. Anesth Analg 2006; 103: 1099-108.

21. Lewis S), Egger M, Sylvester PA, et al. Early enteral feeding versus "nil by mouth" after gastrointestinal surgery: systematic review and meta-analysis of controlled trials. BMJ 2001; 323: 773-6.

22. Han-Geurts IJ, Hop WC, Kok NF, et al. Randomized clinical trial of the impact of early enteral feeding on postoperative ileus and recovery. Br I Surg 2007; 94: 555-61.

23. Lassen K, Kjaeve J, Fetveit T, et al. Allowing normal food at will after major upper gastrointestinal surgery does not increase morbidity: a randomized multicenter trial. Ann Surg 2008; 247: 721-9.

24. Varadhan KK, Lobo DN. A meta-analysis of randomised controlled trials of intravenous fluid therapy in major elective open abdominal surgery: getting the balance right. Proc Nutr Soc 2010; 69: 488-98.

25. Lobo DN, Bostock KA, Neal KR, et al. Effect of salt and water balance on recovery of gastrointestinal function after elective colonic resection: a randomised controlled trial. Lancet 2002; 359: 1812-8.

26. Nisanevich V, Felsenstein I, Almogy G, et al. Effect of intraoperative fluid management on outcome after intraabdominal surgery. Anesthesiology 2005; 103: 25-32.

27. Yang Z, Zheng Q, Wang Z. Meta-analysis of the need for nasogastric or nasojejunal decompression after gastrectomy for gastric cancer. Br J Surg 2008; 95: 809-16.
28. Petrowsky H, Demartines N, Rousson V, et al. Evidence-based value of prophylactic drainage in gastrointestinal surgery: a systematic review and meta-analyses. Ann Surg 2004; 240 : 1074-84.

29. Kawai M, Tani M, Terasawa H, et al. Early removal of prophylactic drains reduces the risk of intra-abdominal infections in patients with pancreatic head resection: prospective study for 104 consecutive patients. Ann Surg 2006; 244: 1-7.

30. Neudecker J, Schwenk W, Junghans T, et al. Randomized controlled trial to examine the influence of thoracic epidural analgesia on postoperative ileus after laparoscopic sigmoid resection. Br J Surg 1999; 86: 1292-5.

31. Baca B, Gonenc M, Hamzaodlu I, et al. Randomized clinical trial comparing epidural anaesthesia and patient-controlled analgesia after laparoscopic segmental colectomy. Br J Surg 2003; 90: 1195-9.

32. Vlug MS, Wind J, Hollmann MW, et al. Laparoscopy in combination with fast track multimodal management is the best perioperative strategy in patients undergoing colonic surgery: a randomized clinical trial (LAFA-study). Ann Surg 2011; 254: 868-75.

33. Smart NJ, White P, Allison AS, et al. Deviation and failure of enhanced recovery after surgery following laparoscopic colorectal surgery: early prediction model. Colorectal Dis 2012; 14: e727-34.

34. Sun J, Li J, Wang J, et al. Meta-analysis of randomized controlled trials on laparoscopic gastrectomy vs. open gastrectomy for distal gastric cancer. Hepatogastroenterology 2012; 59: 1699705.

35. Sato H, Shimada M, Kurita N, et al. Comparison of long-term prognosis of laparoscopy-assisted gastrectomy and conventional open gastrectomy with special reference to D2 lymph node dissection. Surg Endosc 2012; 26: 2240-6.

Received: 20.09.2013, accepted: 17.02.2014. 